\title{
HYBRID INVERSION OF INTERVAL VELOCITIES IN MULTISCALE APPROACH
}

\author{
Rodrigo de S. Santos ${ }^{1}$ and Milton J. Porsani ${ }^{2}$
}

\begin{abstract}
The understanding of the interior of the planet by using the seismic method of reflection requires knowledge of the velocities with which the seismic waves propagate in the subsurface of the Earth. This work presents strategies to obtain the velocity intervals using RMS velocity inversion. Using a hybrid algorithm that combines the Very Fast Simulated Annealing (VFSA) global optimization method and the Fletcher-Reeves local search method, we have sought to reduce the dependence between the accuracy of the results and the model by which the optimization process begins. The main innovative contribution of this study was the development and presentation of the named inversion strategy of multiscale parameters. This technique allows the use of the VFSA method in inversion problems in which the number of variables is significantly large. The hybrid algorithm with multiscale approach was used to solve $1 \mathrm{D}$ and 2D problems, estimating models with high degrees of accuracy, which allowed to confirm the efficiency of the proposed method.
\end{abstract}

Keywords: inversion, parameter multiscale, interval velocity, Very Fast Simulated Annealing, Fletcher-Reeves, hybrid.

RESUMO. 0 entendimento do interior do planeta por meio do método sísmico de reflexão requer o conhecimento das velocidades com que as ondas sísmicas se propagam na subsuperfície da Terra. Este trabalho apresenta estratégias para a obtenção das velocidade intervalares por uso inversão de velocidades RMS. Utilizando um algoritmo híbrido, que combina o método de otimização global Very Fast Simulated Annealing (VFSA), e o método de busca local Fletcher-Reeves, buscou-se reduzir a dependência entre a acurácia dos resultados e o modelo pelo qual o processo de otimização se inicia. A principal contribuição inovadora deste estudo foi 0 desenvolvimento e apresentação da estratégia de inversão nomeada de multiescala de parâmetros. Esta técnica possibilita o uso do método VFSA em problemas de inversão em que o número de variáveis é significativamente grande. 0 algoritmo híbrido com abordagem multiescala foi usado para solucionar problemas 1D e 2D, estimando modelos com elevado grau de acurácia, o que permitiu confirmar a eficiência do método proposto.

Palavras-chave: inversão, multiescala de parâmetros, velocidade intervalar, Very Fast Simulated Annealing, Fletcher-Reeves, híbrido.

\footnotetext{
${ }^{1}$ Centro Universitário SENAI CIMATEC, Research Center in High-performance Processing, Avenida Orlando Gomes, 1845, 41650-010 Salvador, BA, Brazil. Phone: +55(71) 98249-5732 - E-mail: rodrigo_geofisico@hotmail.com

2 Universidade Federal da Bahia (UFBA), Research Center in Geophysics and Geology (CPGG), and National Institute of Science and Technology of Petroleum Geophysics (INCT-GP/CNPq), Rua Barão de Geremoabo, s/n, 40170-115 Salvador, BA, Brazil. Phone: +55(71) 3283-8530; Fax: +55(71) 3283-8501 - E-mail: porsani@ufba.br
} 


\section{INTRODUCTION}

Due to its ability to record mirrorings related to reflectors, located kilometers deep from the surface, the reflection seismic method had become over the years one of the main tools of geophysics applied to hydrocarbon exploration. The understanding of the planet interior through seismic method requires the mapping of the propagate velocities of the elastic waves in the subsurface, i.e., the knowledge of the seismic velocities field. For that reason, the interval velocities determination is a persistent problem in seismic analysis and is critically important for efficient seismic imaging. The most conventional way of the obtained velocities is with Dix equation (Dix, 1955). However, the use of Dix equation is restricted to situations where the data is free from noise, because small variations in the RMS profile cause abrupt and anomalous variations in the obtained results. For that reason, many studies have dealt with the problem of interval velocities with the use of inversion techniques (as: Schultz (1982); Stewart (1984); Rocha Junior \& Porsani (2013); Santana \& Bassrei (2015)). Many of those approaches have limitations such as strong dependence on the initial model, or they need priori information. In this work, improved techniques were developed to obtain interval velocities, by inversion of RMS velocities, which is done independently from the initial model.

Local and global search methods have been consistently applied in solving inverse problems. In this work, we developed a hybrid algorithm that combines the global method Very Fast Simulated Annealing - VFSA (Sen \& Stoffa, 1995), with the Fletcher-Reeves local method - FR (Wright \& Nocedal, 1999), in a multiscale approach in the parameter domain. The combination of local and global methods allowed us to estimate velocity models with high degrees of accuracy without the need of an initial model.

The efficiency and accuracy of inversion methods remain closely linked to the number of inversion parameters. In typical geophysical nonlinear inverse problems, we can easily have thousands of inversion parameters and several secondary maxima. In this work, it is presented a multiscale parameter strategy, which allows a successful estimate of a variety of parameter problems.

\section{THEORY}

In CMP processing, the difference between the zero-offset reflection time $\left(t_{0}\right)$ and the time for another offset is called normal move-out (NMO) difference $\left(\Delta t_{n, N M O}\right)^{3}$. The NMO's effect in a CMP panel make the midpoint reflections hyperbolic, and

\footnotetext{
${ }^{3}$ The term NMO is used for plan reflectors and moveout dip for dip reflectors
}

NMO's correction is the process that removes these effects in a CMP panel (Fig. 1). $\Delta t_{n, N M O}$ is evaluated by:

$$
\Delta t_{n, N M O}=\left[t_{0, n}^{2}+\left(\frac{X}{V_{N M O}}\right)^{2}\right]^{1 / 2}-t_{0, n}
$$

where $X$ represents the offset, $V_{N M O}$ is the NMO's velocity and $t_{0, n}$ the double-time of the zero-offset reflection (Yilmaz, 2001).

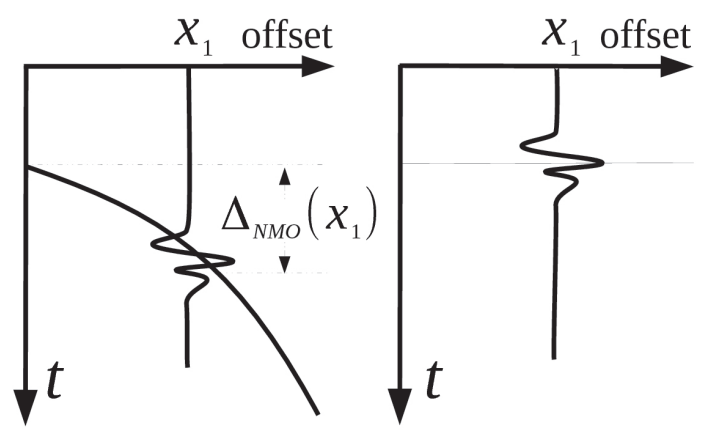

Figure 1 - Demonstrative diagram of the NMO correction application and horizontalization of the reflection hyperbole.

For a medium with a single layer $V_{N M O}$ is this interval measure of velocity. Taner \& Koehler (1969) showed that when the medium is composed of $n$ isovelocity layers with velocities $v_{1}, v_{2}, \ldots, v_{n}$, and double-time between top and bottom of each of them is $t_{1}, t_{2}, \ldots, t_{n}, V_{N M O}$ can be approximated by the Root Mean Square velocity (RMS velocity or $V_{R M S}$ ), and this can be calculated by:

$$
V_{R M S, n}=\sqrt{\frac{1}{t_{0, n}} \sum_{i=n}^{n} v_{i}^{2} t_{i}},
$$

where $t_{0, n}$ is the total double-time between the source and reflection point at the $n$-th layer. In the CMP processing, $V_{R M S, n}$ is obtained by the velocity analysis process as velocities that horizontalizes the reflection's hyperboles in the NMO's correction. In this work, the Eq. (2) was used to obtain $v_{i}$, a nonlinear inverse problem solution, which has as input data the RMS velocities.

Solving the inverse problem means finding the model's parameters $(\mathbf{m})$ when problem's data $(\mathbf{d})$ is known. The inverse problem solution is based on the minimization of the objective function $Q(\mathbf{m})$, where such a function usually measures the energy of the errors between observed data $(\mathbf{d})$ and calculated data $\left(\mathbf{d}_{c a l}\right)$ as shown in the equation below:

$$
Q(\mathbf{m})=\mathbf{e}^{T} \mathbf{e} \quad \text { where } \quad \mathbf{e}=\mathbf{d}-\mathbf{d}_{c a l},
$$


with,

$$
\mathbf{d}_{c a l}=G(\mathbf{m})
$$

or

$\mathbf{d}_{c a l}=\mathbf{G} m \quad$ if $\mathbf{m}$ and $\mathbf{d}$ are LD (linearly dependent).

In the problems where $\mathbf{m}$ and $\mathbf{d}$ are linearly independent (LI), $Q$ (m) represents a rough surface with various local minima (Fig. 2), and for this type of problem the inversion methods are divided in local and global, and each has advantages and disadvantages. The local methods are sensitive to the initial model $\left(\mathbf{m}_{o}\right)$ and converges it to the nearest minimum. Unlike local method, global methods allow the global minimum to be found independent from the initial model. In this work the nonlinear problem was solved by combining the global method Very Fast Simulated Annealing (Sen \& Stoffa, 1995), with the local method Fletcher-Reeves (Wright \& Nocedal, 1999).

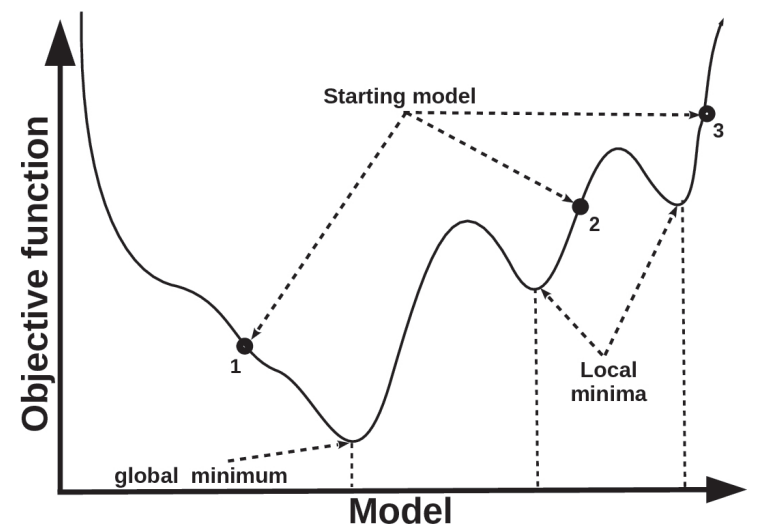

Figure 2 - Objective function of the nonlinear problem with local and global minima. This scheme exemplifies how the local method can estimate least local when the initial model is 2 or 3 (Sen \& Stoffa, 1995).

\section{Fletcher and Reeves Method - FR}

The linear conjugate gradient method was proposed by Hestenes and Stiefel in the 1950s as an iterative method for solving linear systems with positive definite coefficient matrices (Hestenes \& Stiefel, 1952). Fletcher \& Reeves (1964) showed how to extend the conjugate gradient method to nonlinear functions by making two simple changes in it.

First, the nonlinear objective function gradient is calculated at each iteration by:

$$
\nabla Q_{k+1}=\nabla Q\left(\mathbf{m}_{k+1}\right)
$$

Thus, at each iteration the model is updated by (6):

$$
\mathbf{m}_{k+1}=\mathbf{m}_{k}+\alpha_{k} \mathbf{v}_{k},
$$

where $\mathbf{v}_{k}$ is calculated by:

$$
\mathbf{v}_{k+1}=-\nabla Q_{k+1}+\beta_{k+1}^{F R} \mathbf{v}_{k},
$$

with

$$
\beta_{k+1}^{F R}=\frac{\nabla Q_{k+1}^{T} \nabla Q_{k+1}}{\nabla Q_{k}^{T} \nabla Q_{k}} .
$$

Second, for the computation of the step length $\alpha_{k}$, we need to perform a line search which identifies as an approximate minimum of the nonlinear function $Q$ along $\mathbf{v}_{k}$ (Wright \& Nocedal, 1999). The step length $\alpha_{k}$ need to satisfy the strong Wolfe conditions written here as:

$$
\begin{gathered}
Q\left(\mathbf{m}_{k}+\alpha_{k} \mathbf{v}_{k}\right) \leq Q\left(\mathbf{m}_{k}\right)+C_{1} \alpha_{k} \nabla Q\left(\mathbf{m}_{k}\right)^{T} \mathbf{v}_{k}, \\
\left|\nabla Q\left(\mathbf{m}_{k}+\alpha_{k} \mathbf{v}_{k}\right)^{T} \mathbf{v}_{k}\right| \leq-C_{2} \nabla Q\left(\mathbf{m}_{k}\right)^{T} \mathbf{v}_{k},
\end{gathered}
$$

where $0<c_{1}<c_{2}<1 / 2$ (Wright \& Nocedal, 1999). The Fletcher-Reeves Method is a local search method, and the referred estimated model represents the closest minimum to the initial model (Fig. 2).

\section{Very Fast Simulated Annealing - VFSA}

The Very Fast Simulated Annealing method permits to find the global minimum of the objective function (Sen \& Stoffa, 1995). It is a variant of the Simulated Annealing, which allows narrowing the search interval at each iteration, resulting in faster convergence. From an initial model $\mathbf{m}_{k}$, updating occurs through Eq. (10), where each parameter $m_{k}^{i}$ is disturbed by a factor $y_{i}$, generated randomly by the equation below (Sen \& Stoffa, 1995):

$$
m_{i}^{k+1}=m_{i}^{k}+y_{i}\left(m_{i}^{\max }-m_{i}^{\min }\right) .
$$

The parameter $y_{i}$ is generated from the following distribution:

$$
\begin{aligned}
g_{T}(y) & =\prod_{i=1}^{N M} \frac{1}{2\left(\left|y_{i}\right|+T_{i}\right) \ln \left(1+\frac{1}{T_{i}}\right)} \\
& =\prod_{i=1}^{N M} g_{T_{i}}\left(y_{i}\right) .
\end{aligned}
$$

Thus a random number $u_{i}$ drawn from a uniform distribution $u[0,1]$ can be mapped into the above distribution with the formula:

$$
y_{i}=\operatorname{sgn}\left(u_{i}-\frac{1}{2}\right) T_{i}\left[\left(1+\frac{1}{T_{i}}\right)^{\left|2 u_{i}-1\right|}-1\right],
$$

with $m_{i}^{\max }$ and $m_{i}^{\min }$ representing the bounds of the model, and $u_{i}$ is generated randomly. Ingber (1989) showed that the 
overall minimum is obtained statistically using the cooling criterion shown in Eq. (13), where the temperature $T_{i}$ is reduced at each iteration with:

$$
T_{i}(k)=T_{0 i} e^{-C_{i} k^{1 / N M}} .
$$

The model generated by Eq. (10) will be taken as the current model based on the criterion of metropolis (Metropolis et al., 1953). The statistical character of generation and acceptance allows the algorithm to surpass from local minimum.

\section{Hybrid Optimization Methods}

Local and global optimization algorithms are used commonly in geophysical data inversion. Each type of algorithm has unique advantages and disadvantages. Chunduru et al. (1997) showed that hybrid algorithms are computationally more efficient than conventional global optimization methods. Hybrid search algorithms have the potential to make use of the important features of both global and local algorithms, as they do not require a good starting solution; are computationally less expensive compared to global algorithms and can obtain good models with poor starting solutions (Chunduru et al., 1997). In this work, we combine the local FR method with a global VFSA approach to solving problems of geophysical interest. The method structure is FR at the end of the VFSA, where the VFSA gets the starting model for FR. The FR combination with VFSA is named of the hybrid FletcherReeves (HFR).

\section{RESULTS}

Treating the velocity intervals as a solution to the inverse problem, we can see, by comparing Eqs. (4) and (2):

$$
\begin{aligned}
\mathbf{m} & =\left[v_{1} \ldots v_{N}\right]^{T} \\
\mathbf{d} & =\left[\mathrm{V}_{R M S, 1} \ldots \mathrm{V}_{R M S, M}\right]^{T},
\end{aligned}
$$

where the data vector $(\mathbf{d})$ is represented by the RMS velocity profiles samples set, and the model parameters $(\mathbf{m})$ is represented by the interval velocity profiles samples. We will show the result of the application of the HFR method in the solution of this problem.

The method performance was evaluated using the criterion of relative RMS deviation of the data $\left(\epsilon_{R M S}^{\mathbf{d}}\right)$ and of the model parameter $\left(\epsilon_{R M S}^{\mathbf{m}}\right)$, defined as:

$$
\epsilon_{R M S}^{\mathbf{d}}=\frac{\sqrt{\sum_{i=1}^{N}\left(d_{i}-d_{i}^{c a l}\right)^{2}}}{\sqrt{\sum_{i=1}^{N}\left(d_{i}\right)^{2}}},
$$

and,

$$
\epsilon_{R M S}^{\mathbf{m}}=\frac{\sqrt{\sum_{k=1}^{M}\left(m_{k}^{\text {ver }}-m_{k}^{\text {est }}\right)^{2}}}{\sqrt{\sum_{k=1}^{M}\left(m_{k}^{\text {true }}\right)^{2}}}
$$

with $\mathbf{d}$ and $\mathbf{d}^{c a l}$ representing the observed and calculated data vectors, respectively, and $\mathbf{m}^{\text {true }}$ and $\mathbf{m}^{\text {est }}$ the true and estimated parameter models.

\section{M1 Model}

The M1 model represents an interval time velocities $(v)$ profile, with $N$ samples separated by $4 \mathrm{~ms}\left(4.10^{-3} \mathrm{~s}\right)$, where each velocity represents a model's parameter that is calculated by the equation:

$$
\begin{gathered}
v_{i}=800 \times\left[3-\sin \left(\frac{i \times 6,5231}{N}\right)\right] \mathrm{m} / \mathrm{s} \\
\text { with } i \epsilon[1, \ldots, N] .
\end{gathered}
$$

The M1 model assumed four different configurations: $N=10$, $N=30, N=50$ and $N=100$. For each M1 model configuration, an RMS velocity profile was generated by Eq. (2) with $t i=2 \mathrm{~ms}$ and $M=2 N$.

Using the HFR inversion method we estimate the model parameters' vector $\left(v_{i}\right)$, starting from an initial constant model $V_{0}=2400 \mathrm{~m} / \mathrm{s}$. Figures 3(a), 3(b), 3(c) and 3(d) show the results for the configurations $N=10, N=30, N=50$ and $N=100$, were the true, estimated and initial models are shown. Table 1 shows the RMS errors of this data.

The analysis of the Figures 3(a), 3(b), 3(c) and 3(d) together with analysis of Table 1, makes it possible to conclude that with the inversion parameters number's increase, there is a significant reduction in the accuracy of the results. It occurs because, with the increase in the parameters number, there is an increase in the model solution space, and consequently it directly affects the VFSA stochastic method's efficiency. Thus, the number of inversion parameters is a limiting factor for the use of probabilistic methods, and is the main reason why this class of methods is not used in solving problems with a high number of inversion parameters, such as FWI (Full Waveform Inversion) for example. In order to solve this problem, we developed a technique called Parameter Multiscale Approach, which will be presented below.

\section{Multiscale Approach}

The parameters multiscale approach is applied in problems where the number of inversion parameters is large enough to compromise the results. This technique consists of resolving the problem 


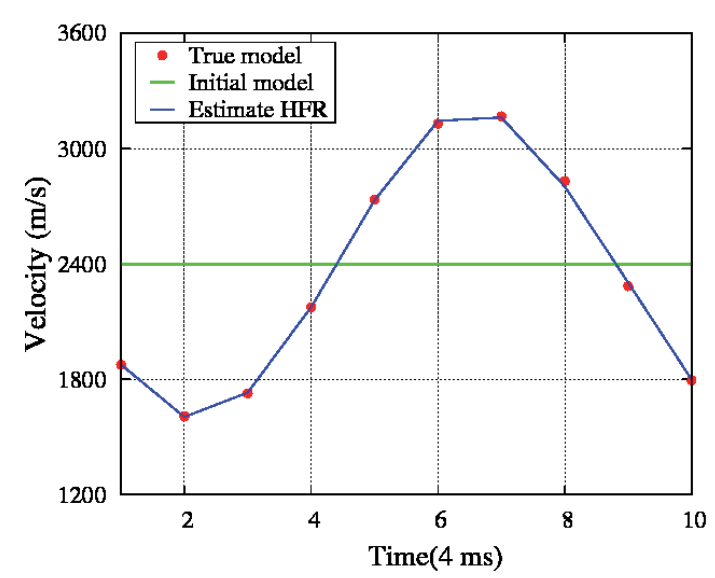

(a) Velocity profile M1 model with $N=10$.

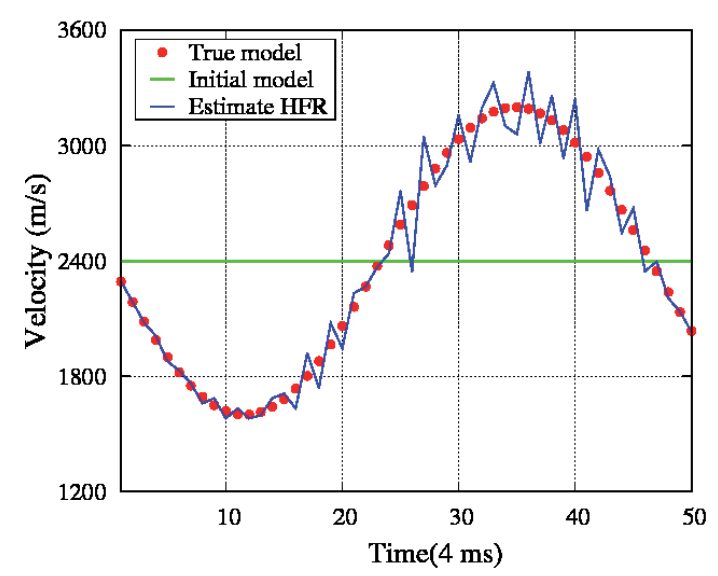

(c) Velocity profile M1 model with $N=50$.

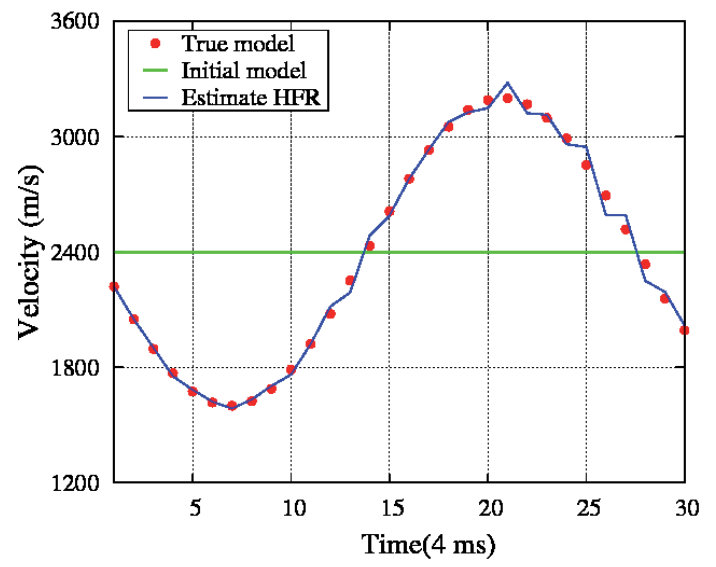

(b) Velocity profile M1 model with $N=30$.

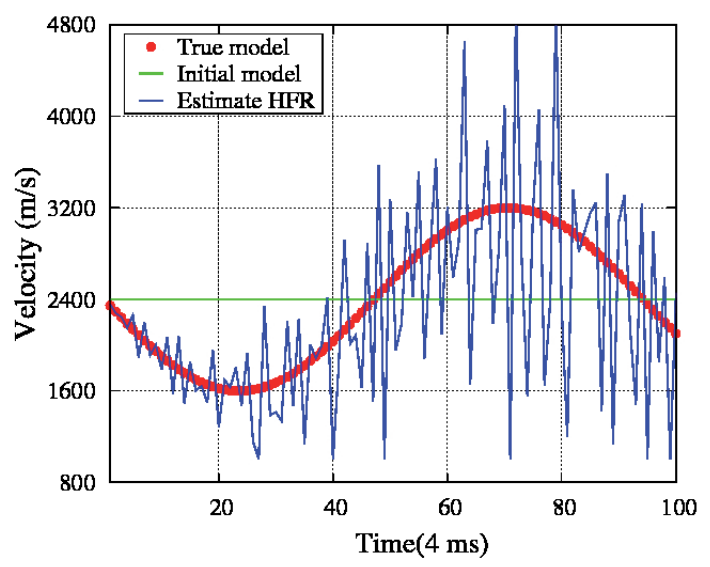

(d) Velocity profile M1 model with $N=100$.

Figure 3 - Interval velocity profiles as a function of time. The figures from (a) to (d) show the true initial and estimated velocities for the M1 model with the different values of $N$. With $N=10$ in (a) $N=30$ in (b) $N=50$ in (c) and $N=100$ in (d).

Table 1 - RMS errors calculated for M1 model with $N=10,30,50$ and 100

\begin{tabular}{|c|c|c|}
\hline \multicolumn{3}{|c|}{ RMS errors of the M1 model } \\
\hline & $\epsilon_{R M S}^{\mathbf{d}}$ & $\epsilon_{R M S}^{\mathbf{m}}$ \\
\hline$N=10$ & $7.35 \times 10^{-4}$ & $5.17 \times 10^{-3}$ \\
\hline$N=30$ & $6.29 \times 10^{-4}$ & $7.05 \times 10^{-3}$ \\
\hline$N=50$ & $8.29 \times 10^{-4}$ & $1.18 \times 10^{-2}$ \\
\hline$N=100$ & $4.41 \times 10^{-3}$ & $4.81 \times 10^{-2}$ \\
\hline
\end{tabular}

in successive rounds, using different scales in the solution space. In the first round, the number of inversion parameters is much smaller than the number of model parameters, as are the minima of the solution space, increasing in number at each round. The model obtained in the previous round is taken as the initial model of the next round. This process repeats itself until the problem is completely solved.

The physical principle behind the technique is the same as one used by Bunks et al. (1995) in the frequency multiscale used in FWI. When the process starts with a number of parameters 
much lower than the number of true parameters, it is possible to recover larger structures, however, without great detail. As the number of parameters increases at each round, the obtained model shows greater detail and is closer to the true model.

In short, this approach is done in three steps:

- Step 1 - to assume that the velocity model to be obtained is represented by a profile with $N$ different parameters. In this step, a windowing of this profile is created and divided it into cells.

- Step 2 - the problem is solved by assuming that all parameters of the same cell are represented by a single value.

- Step 3 - each cell is re-divided, and step 2 is executed again by taking the model estimated in the previous round as the initial model.

Using the multiscale approach, the problem of the M1 model was solved again with two new configurations, $N=300$ and $N=1000$, and again starting with a constant initial model with $V_{0}=2400$. These results are shown in Figures $4(\mathrm{a})$ and 4(b), and RMS errors are shown in Table 2. Figures 4(c) and 4(d) show the function evolution at each round of the multiscale. These results demonstrate the multiscale method's efficiency in solving the velocity problem.

\section{M2 Model - Marmousi}

The Marmousi model was created by the Institut Français du Pétrole (IFP) in 1988, and synthetic seismic data were generated from this model (Brougois et al., 1990). The geometry of this model is based on a profile through the North Quenguela trough in the Cuanza basin. Figure 5(a) shows this model, whose dimensions are $375 \times 369$. That model is performed at one-dimensional and two-dimensional modelling and inversion. Using the 375 velocity samples from the 100-th column of the Marmousi model, a RMS profile with 125 samples was modelled. This RMS profile has been inverted, generating the interval profile with 375 samples, in a typical sub-determined problem, using the multiscale approach shown in the Figure 5(b), with

$$
\epsilon_{R M S}^{\mathbf{d}}=9,02 \times 10^{-5} \quad \text { and } \quad \epsilon_{R M S}^{\mathbf{m}}=7,13 \times 10^{-2} .
$$

Figure 5(c) shows the function evolution for each multiscale round.

This experiment was repeated with all 369 columns of the model, and the inverted results were interpolated, generating the estimated 2D model shown in the Figure 5(d).

\section{M3 Model - Ray Tracing Seismic Modeling}

The M3 model shown in Figure 6(a) was subjected to a simulated ray tracing modelling, according to the parameters of Table 3.

The generated seismic line was organized in CMPs and subjected to velocity analysis, generating a stacking velocities field which approximates the $V_{R M S}$ field. From this field, the RMS profile corresponding to CMP 146 was selected, which has 1160 $V_{R M S}$ samples spaced $2 \mathrm{~ms}$ apart. This profile was inverted resulting in the interval profile shown in Figure 6(b) with 580 samples separated by $4 \mathrm{~ms}$ of time. The Figure 6(c) shows the evolution of the function to each round of the multiscale.

By repeating the experiment performed on the CMP 146, in each of the 497 CMP's of the data, the 2D inverted section was obtained through the interpolation of these profiles, as shown the Figure 6(d).

\section{CONCLUSIONS}

In this work, the VFSA methods with FR were combined in the search for the solution of the nonlinear inverse problem. The FR action is after the VFSA, so the hybrid method's sucess depends on the VFSA's sucess. Generally, the use of the VFSA method makes it possible to estimate the overall solution of the inverse problem, even when a good initial estimate of the solution is not known. One of the main barriers to the use of this type of method is related to the number of inversion parameters.

When the problem has a few variables, the method behaves effectively and efficiently, as shown in the Figure 3(a). However, as the number of variables grows, it becomes increasingly difficult to perform inversion (Fig. 3(b) and 3(c)), so that when the number of parameters is significantly large, it becomes impracticable to use this type of method (Fig. 3(d)). In this work, this problem was solved using the multiscale parameter approach, as seen in Figure 4(a) and 4(b), even in situations where there are many variables to determine, it is possible to obtain relative accuracy in the problem's solution. As in the M1 model, the use of the multiscale in the Marmousi model provided the problem's solution with a high degree of accuracy, which validates the proposed method's success.

The multiscale approach was developed and used successfully to solve the problem of interval velocities, but it is believed that this approach is applicable to different problems where it is desired to estimate a large number of variables with stochastic methods.

The velocity estimation performed on the M1 and M2 models were performed with noise-free data in situations of over- 


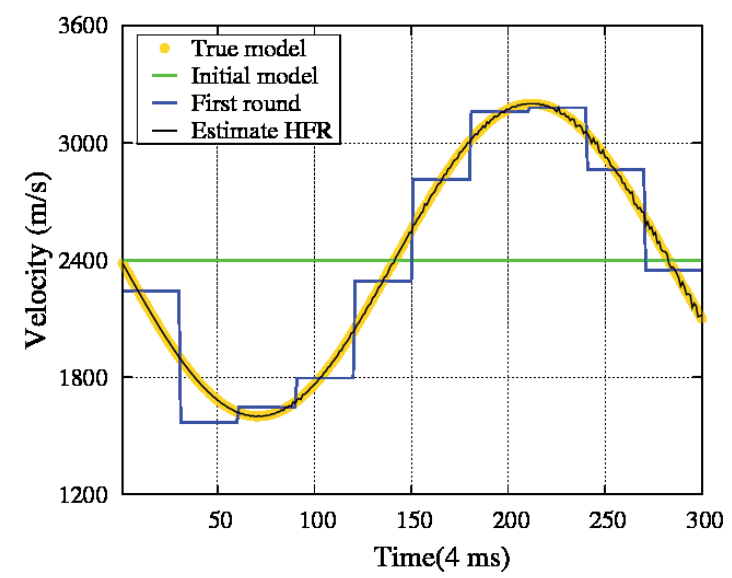

(a) Velocity profile M1 model with $N=300$.

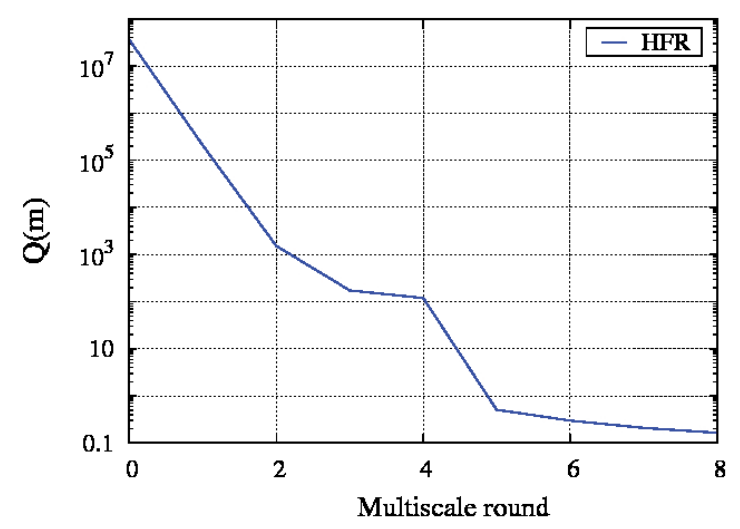

(c) Objective function M1 Model with $N=300$.

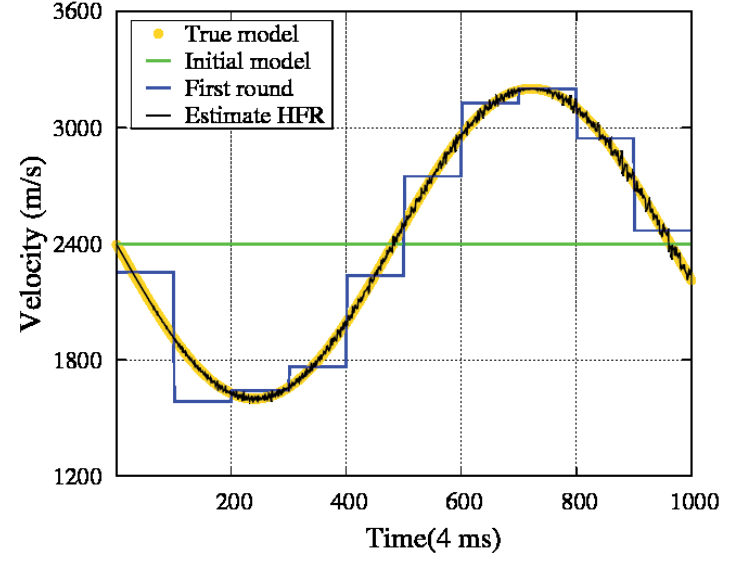

(b) Velocity profile M1 model with $N=1000$

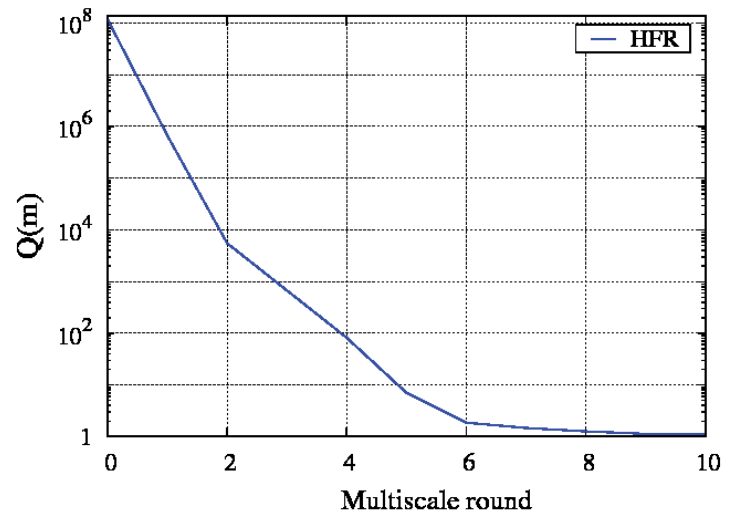

(d) Objective function M1 Model with $N=1000$.

Figure 4 - Interval velocity profiles as a function of time. The figures from (a) to (d) show the true, initial and estimated velocities with the multiscale approach, in M1 model with (a) $N=300$ and (b) $N=1000$. In (c) and (d) the evolution of the objective function versus multiscale rounds is shown.

Table 2 - RMS errors calculated for M1 model with $N=300$ and 1000, with the multiscale approach.

\begin{tabular}{|c|c|c|}
\hline \multicolumn{3}{|c|}{ RMS errors of the M1 model with multiscale } \\
\hline & $\epsilon_{R M S}^{\mathrm{d}}$ & $\epsilon_{R M S}^{\mathrm{m}}$ \\
\hline$N=300$ & $1.38 \times 10^{-6}$ & $1.08 \times 10^{-5}$ \\
\hline$N=1000$ & $2.43 \times 10^{-6}$ & $2.57 \times 10^{-5}$ \\
\hline
\end{tabular}

Table 3 - Information from the seismic line modeled by the Cshot ray tracing

\begin{tabular}{|c|c|c|c|}
\hline \multicolumn{5}{|c|}{ Seismic modeling parameters in the M3 model } \\
\hline Shots amount total & 200 & CMP's amount total & 497 \\
\hline Channels amount & 96 & CMP fold & 48 trace \\
\hline Distance between channels & $51(\mathrm{ft})$ & Number of samples & 1300 \\
\hline Registration time & $2.6 \mathrm{sec}$ & Sampling interval & $2 \mathrm{~m}$ \\
\hline
\end{tabular}




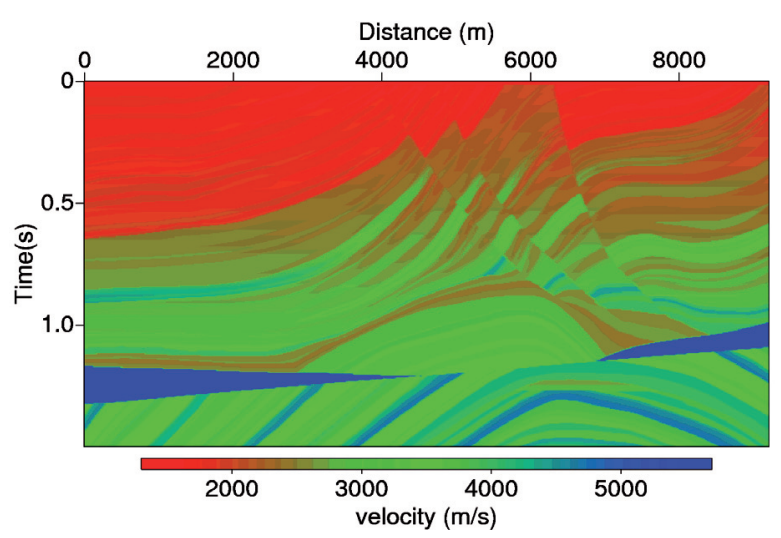

(a) Marmousi Model.

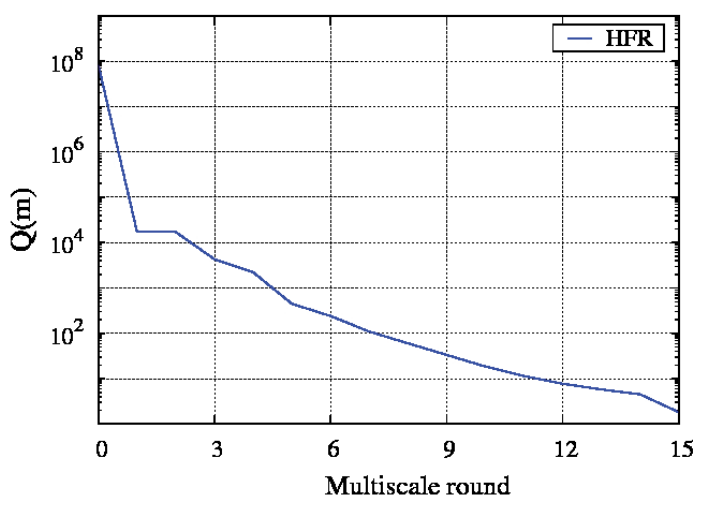

(c) Objective function.

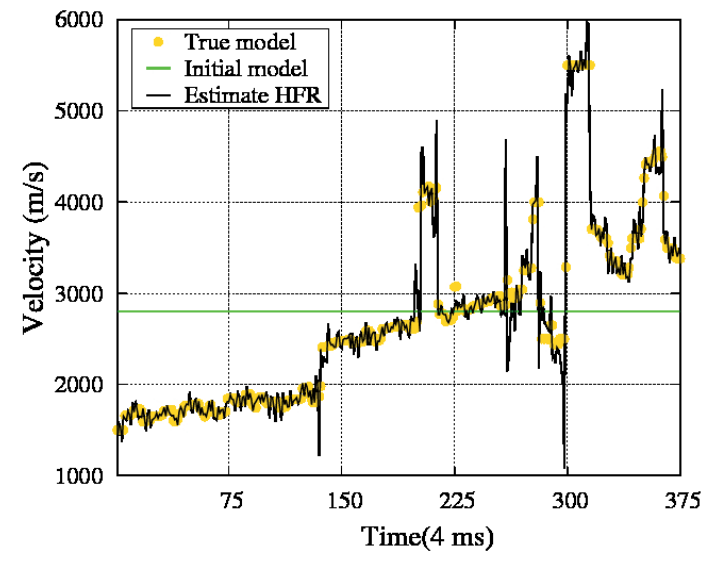

(b) Marmousi $1 \mathrm{D}$ inversion.

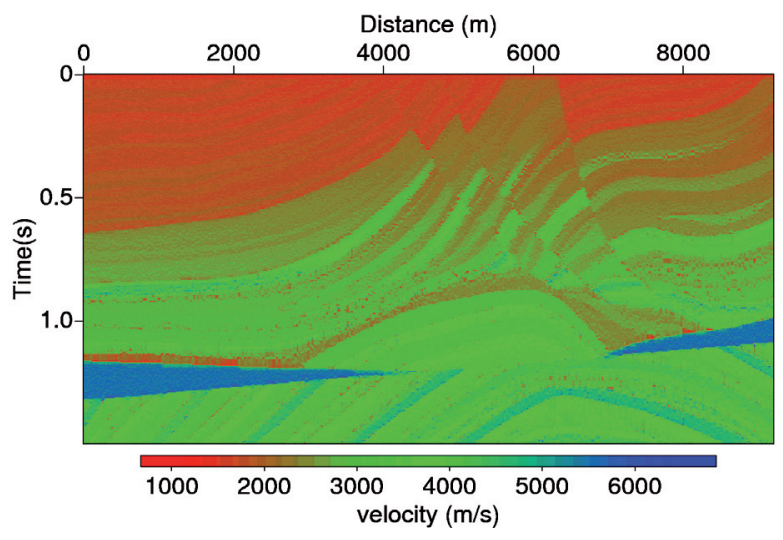

(d) Marmousi 2D inversion.

Figure 5 - 1D and 2D models estimated by the hybrid multiscale approach method - Marmousi.

determined and under-determined problems, respectively, resulting in an estimate with a high degree of accuracy. In the M3 model, seismic modelling was performed, and the $V_{R M S}$ was obtained through velocities analysis, which resulted in noise contaminated data. This set of residues present in the data had a significant influence on the estimation of the model; it can be concluded that the main limitation of obtaining interval velocities with a high degree of accuracy by such approach is related to the difficulty of accurately estimating $V_{R M S}$.

Therefore, the need to explore other ways of obtaining $V_{R M S}$ is left as a proposal for future works, such as the use of highresolution velocity spectra.

\section{ACKNOWLEDGMENTS}

The authors wish to express their gratitude to the INCT-GP, CNPq, Brazil, for financial support.

\section{REFERENCES}

BROUGOIS A, BOURGET M, LAILLY P, POULET M, RICARTE P \& VERSTEEG R. 1990. Marmousi, model and data. In: EAEG WorkshopPractical Aspects of Seismic Data Inversion. Copenhagen, pp. 5-16.

BUNKS C, SALECK FM, ZALESKI S \& CHAVENT G. 1995. Multiscale seismic waveform inversion. Geophysics, 60(5): 1457-1473.

CHUNDURU RK, SEN MK \& STOFFA PL. 1997. Hybrid optimization methods for geophysical inversion. Geophysics, 62(4): 1196-1207.

DIX CH. 1955. Seismic velocities from surface measurements. Geophysics, 20(1): 68-86.

FLETCHER R \& REEVES CM. 1964. Function minimization by conjugate gradients. The Computer Journal, 7(2): 149-154.

HESTENES MR \& STIEFEL E. 1952. Methods of conjugate gradients for solving linear systems. Journal of Research of the National Bureau of Standards, NBS, 49(6): 409-436. 


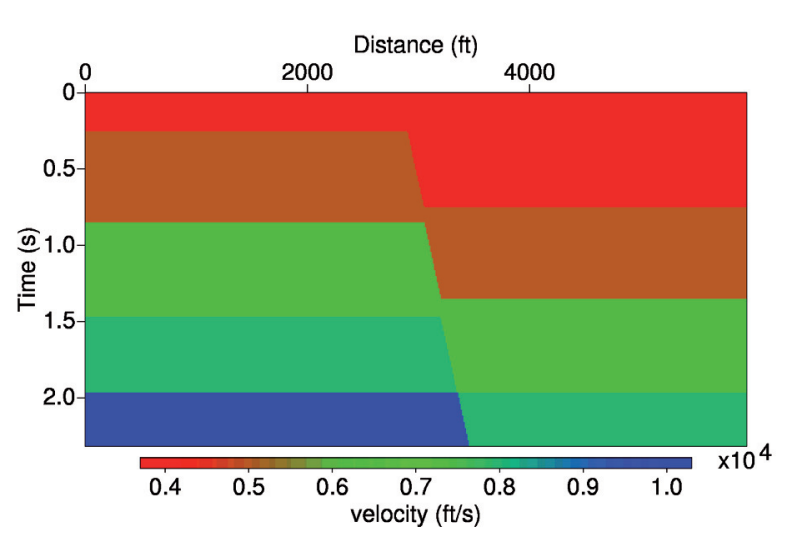

(a) Cshot Model.

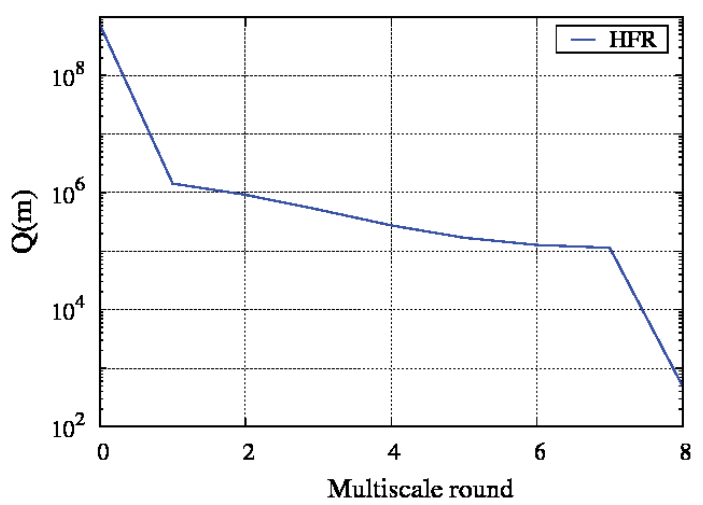

(c) Cshot objective function

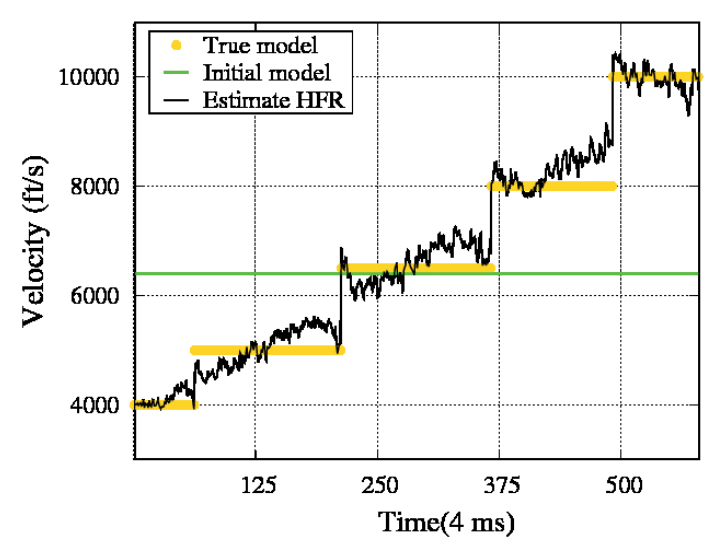

(b) Cshot $1 \mathrm{D}$ inversion.

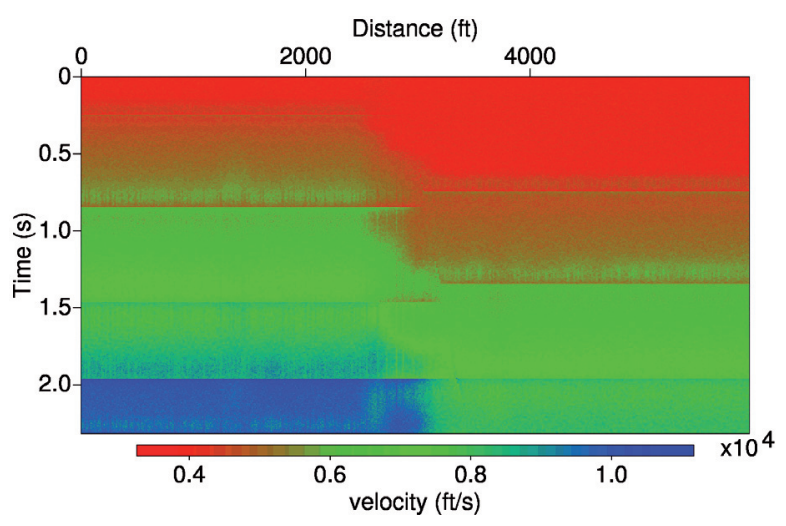

(d) Cshot 2D inversion.

Figure 6-1D and 2D models estimated by the hybrid multiscale approach method - Cshot model.

INGBER L. 1989. Very fast simulated re-annealing. Mathematical and Computer Modelling, 12(8): 967-973.

METROPOLIS N, ROSENBLUTH AW, ROSENBLUTH MN, TELLER AH \& TELLER E. 1953. Equation of state calculations by fast computing machines. The Journal of Chemical Physics, 21(6): 1087-1092.

ROCHA JUNIOR DC \& PORSANI MJ. 2013. Non-linear inversion of interval velocities. In: 13th International Congress of the Brazilian Geophysical Society \& EXPOGEF, Rio de Janeiro, Brazil, 26-29 August2013, pp. 1242-1246. Brazilian Geophysical Society. CD-ROM.

SANTANA TG \& BASSREI A. 2015. Inversion of interval velocities: Application to a geological model from a pre-salt area. Brazilian Journal of Geophysics, 33(1): 19-27.

SCHULTZPS. 1982. A method for direct estimation of interval velocities.
Geophysics, 47(12): 1657-1671.

SEN MK \& STOFFA PL. 1995. Global optimization methods in geophysical inversion. Cambridge University Press. 280 pp.

STEWART R. 1984. VSP interval velocities from traveltime inversion. Geophysical Prospecting, 32(4): 608-628.

TANER MT \& KOEHLER F. 1969. Velocity spectra digital computer derivation applications of velocity functions. Geophysics, 34(6): 859881.

WRIGHT S \& NOCEDAL J. 1999. Numerical optimization. Springer Science, 35: 1-664.

YILMAZ 0. 2001. Seismic data analysis: processing, inversion and interpretation of seismic data. SEG. 2027 pp. 